PREPARED FOR THE U.S. DEPARTMENT OF ENERGY, UNDER CONTRACT DE-AC02-76CH03073

PPPL-3699

PPPL-3699

UC-70

Kinetic Alfvén Waves at the Magnetopause-Mode Conversion,

Transport and Formation of LLBL

by

Jay R. Johnson and C.Z. Cheng

May 2002

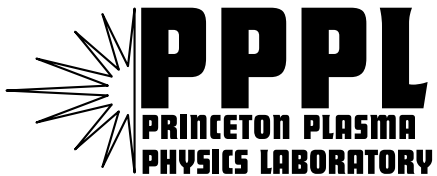

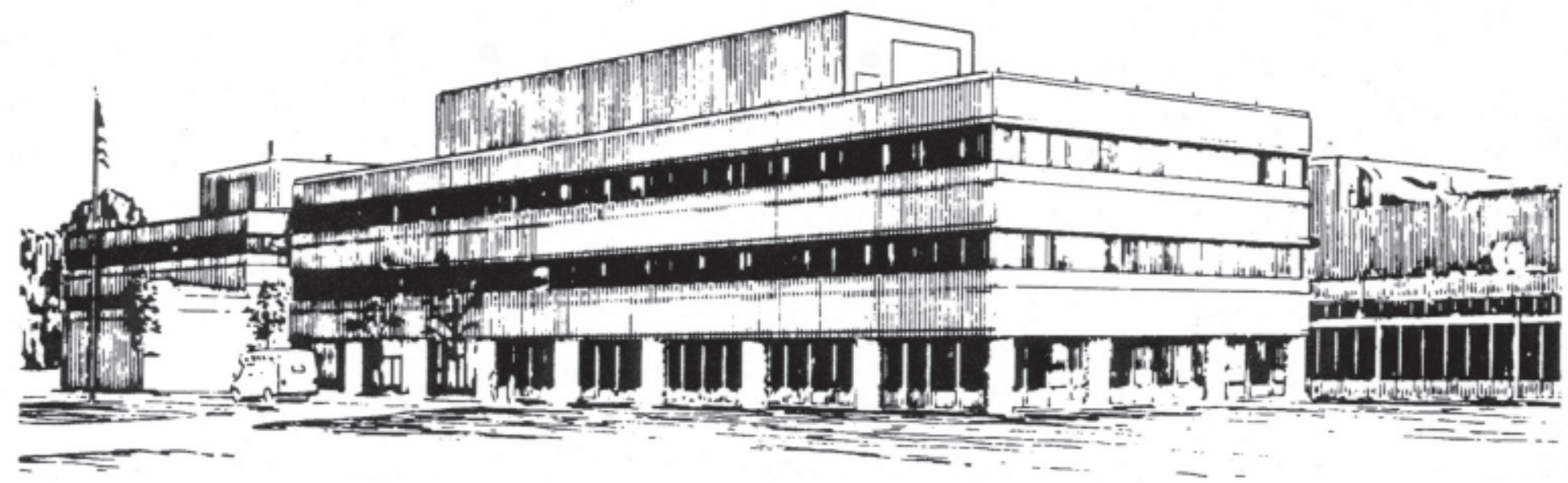

PRINCETON PLASMA PHYSICS LABORATORY PRINCETON UNIVERSITY, PRINCETON, NEW JERSEY 


\section{PPPL Reports Disclaimer}

This report was prepared as an account of work sponsored by an agency of the United States Government. Neither the United States Government nor any agency thereof, nor any of their employees, makes any warranty, express or implied, or assumes any legal liability or responsibility for the accuracy, completeness, or usefulness of any information, apparatus, product, or process disclosed, or represents that its use would not infringe privately owned rights. Reference herein to any specific commercial product, process, or service by trade name, trademark, manufacturer, or otherwise, does not necessarily constitute or imply its endorsement, recommendation, or favoring by the United States Government or any agency thereof. The views and opinions of authors expressed herein do not necessarily state or reflect those of the United States Government or any agency thereof.

\section{Availability}

This report is posted on the U.S. Department of Energy's Princeton Plasma Physics Laboratory Publications and Reports web site in Fiscal Year 2002. The home page for PPPL Reports and Publications is: http://www.pppl.gov/pub_report/

DOE and DOE Contractors can obtain copies of this report from:

U.S. Department of Energy

Office of Scientific and Technical Information

DOE Technical Information Services (DTIS)

P.O. Box 62

Oak Ridge, TN 37831

Telephone: (865) 576-8401

Fax: (865) 576-5728

Email: reports@adonis.osti.gov

This report is available to the general public from:

National Technical Information Service

U.S. Department of Commerce

5285 Port Royal Road

Springfield, VA 22161

Telephone: 1-800-553-6847 or

(703) 605-6000

Fax: (703) 321-8547

Internet: http://www.ntis.gov/ordering.htm 


\title{
Kinetic Alfvén Waves at the Magnetopause-Mode Conversion, Transport and Formation of LLBL
}

\author{
Jay R. Johnson and C. Z. Cheng \\ Princeton University, Plasma Physics Laboratory, Princeton, NJ
}

\begin{abstract}
At the magnetopause, large amplitude, low frequency (ULF), transverse MHD waves are nearly always observed. These waves likely result from mode conversion of compressional MHD waves observed in the magnetosheath to kinetic Alfven waves at the magnetopause where there is a steep gradient in the Alfven velocity [Johnson and Cheng, Geophys. Res. Lett., 24, 1423, (1997)]. The mode conversion process can explain the following wave observations typically found during satellite crossings of the magnetopause: (1) a dramatic change in wave polarization from compressional in the magnetosheath to transverse at the magnetopause, (2) an amplification of wave amplitude at the magnetopause, (3) a change in Poynting flux from crossfield in the magnetosheath to field-aligned at the magnetopause, and (4) a steepening in the wave power spectrum at the magnetopause. We examine magnetic field data from a set of ISEE1, ISEE2, and WIND magnetopause crossings and compare with the predictions of theoretical wave solutions based on the kinetic-fluid model with particular attention to the role of magnetic field rotation across the magnetopause. The results of the study suggest a good qualitative agreement between the observations and the theory of mode conversion to kinetic Alfven waves. Because mode converted kinetic Alfven waves readily decouple particles from the magnetic field lines, efficient quasilinear transport $\left(D \sim 10^{9} \mathrm{~m}^{2} / \mathrm{s}\right)$ can occur. Moreover, if the wave amplitude is sufficiently large $\left(B_{\text {wave }} / B_{0}>0.2\right)$ stochastic particle transport also occurs. This wave induced transport can lead to significant heating and particle entry into the low latitude boundary layer across closed field lines.
\end{abstract}

\section{INTRODUCTION}

Boundary layers are nearly always observed on the Earthward edge of the magnetopause. It is commonly believed that reconnection plays the dominant role in the formation of these boundary layers as evidenced by D-shaped ion distributions, counterstreaming plasma jets, and anisotropies in the electron distribution commonly found in the boundary layer [Phan, 2001; Fuselier, 2001]. However, it is not uncommon to find magnetopause crossings with a typical boundary layer density profile in the absence of these active reconnection signatures [Phan, 2001]. Such observations are suggestive that another physical transport process may be responsible for the formation of the boundary layer in those cases. One such possibility is wave-induced transport. 
In the remainder of this paper we will: (a) present a magnetopause crossing which illustrates the typical magnetopause wave spectrum, (b) identify the characteristics of the wave spectrum and how they relate to kinetic Alfvén waves, (c) present typical kinetic Alfvén wave structure at the magnetopause, (d) examine the role of kinetic Alfvén waves in plasma transport and heating at the magnetopause.

\section{Wave Observations at the Magnetopause}

The magnetopause crossing detected by WIND on December 12, 1996 and shown in Figure Fig. 1 is a good example of a boundary layer crossing in which there are no obvious signatures of reconnection [Phan, 2001]. During this period WIND was passing from the flank magnetosheath into the magnetosphere $\left(X_{\text {gse }} \sim 0\right.$, $Y_{\text {gse }} \sim 15 R_{E}, Z_{\text {gse }} \sim 0$ ). Around 19:00 UT the magnetic field vector rotated by $60^{\circ}$ due to a rotation of the solar wind magnetic field Phan et al. [1997] and the density began to drop gradually. The outer edge of the magnetopause current layer is marked by a distinct magnetic field rotation at 19:20 UT. The satellite moved into the low-latitude boundary layer marked by a rapid density drop at about 19:55 UT and into the plasma sheet at 20:45 UT. The Alfvén velocity increased gradually by a factor of 5 during the magnetopause crossing and the magnetic field vector rotated by around $50^{\circ}$. While in the magnetosheath proper (prior to 19:20 UT), the wave power is mainly in the compressional component as evidenced in the large fluctuations of $B_{t o t}$. The plasma $\beta$ for this period is moderate (around 2) and the pressure anisotropy is not very large [see Fig. 2 from Phan et al. [1997]], suggestive that the waves are compressional Alfvén waves rather than mirror modes. The compressional wave activity continues through the magnetopause even though the plasma $\beta$ gradually drops below unity between 19:20 and 19:55 UT. As the density gradually decreases and the magnetic field increases, the transverse component of the magnetic field fluctuations becomes dominant (note the marked increase in wave activity in the individual GSM Cartesian components of the magnetic field while $B_{t o t}$ fluctuations remain roughly the same). The enhanced transverse wave activity persists all the way into the low-latitude boundary layer from (20:00 UT to 20:45 UT). This magnetopause crossing is typical when compressional wave activity is found in the magnetosheath.

Figure 1

The change in wave polarization from the magnetosheath to the magnetosphere is clearly seen in the power spectrogram for the crossing. Power spectra are 
obtained for all magnetic field components and the magnetic field magnitude and are used to obtain the compressional and transverse magnetic field spectrum. In Plate 1 we show the wave power spectral density of compressional, $P_{\|}$, and transverse, $P_{\perp}$, magnetic fluctuations as well as the fraction of wave power in the transverse magnetic field component. A broad band of waves is found in the $10-100 \mathrm{mHz}$ frequency range. In the magnetosheath, the waves are primarily compressional, but at the magnetopause, where the density and magnetic field gradients are found, the transverse component is dominant in the same frequency range. The compressional magnetic field spectra remain approximately the same from the magnetosheath into the magnetopause with an eventual cutoff as WIND moved through the low-latitude boundary layer into the plasma sheet at 20:45 UT. In the magnetosheath, the transverse component appears in the same frequency range and is well correlated with the compressional component. However, at the magnetopause there is a dramatic increase in the transverse power spectrum as evidenced in the lower panel of Plate 1. Note that the amplification (ratio of spectral density at the magnetopause to that of the magnetosheath) of the transverse spectrum falls off as frequency increases.

Plate 1

In fact, ultra-low frequency (ULF) waves (with frequencies below $500 \mathrm{mHz}$ ) dominate the spectrum of nearly every magnetopause crossing [Perraut et al., 1979; Rezeau et al., 1993; Song et al., 1993c; Song, 1994; Phan and Paschmann, 1996, and references therein]. It has been suggested that these waves are associated with mode conversion of MHD waves in the magnetosheath to kinetic Alfvén waves (KAWs) at the magnetopause near a field line resonance location [Lee et al., 1994; Belmont et al., 1995; De Keyser et al., 1999]. The mode conversion process can explain (a) a change in wave polarization at the magnetopause and (b) the amplification of the transverse magnetic field component by an order of magnitude [Johnson and Cheng, 1997b].

Because the interplanetary magnetic field changes orientation frequently, it is possible to characterize the magnetopause wave activity as a function of magnetic shear (defined to be the angle between the magnetic field in the magnetosheath and the magnetic field on the magnetospheric side of the magnetopause). Because the mode conversion process exhibits a strong dependence on this magnetic field rotation theory/data comparisons of expected wave signatures can be used effectively as a test of the mode conversion process. Data for the comparison was taken from the ISEE1, 
ISEE2 and WIND spacecrafts. Thirteen magnetopause crossings were considered where sizeable compressional magnetic field fluctuations were found in the magnetosheath. These cases provided coverage of the magnetic rotation angle from 0 to 180 degrees. Windowed magnetic power spectra were obtained for parallel and transverse magnetic fluctuations during each crossing. The magnetosheath and magnetopause spectra were compared for each crossing to quantify the wave amplification for each crossing. The wave amplification factor, $P_{\perp m p} / P_{\perp m s h}$, where $P_{\perp m s h}$ and $P_{\perp m p}$ refer to average values of the power spectral density of $\delta \mathbf{B}_{\perp}$ in the magnetosheath and magnetopause respectively, is shown in Fig. 2 for these magnetopause crossings for frequencies of 25 and $50 \mathrm{mHz}$. From the study [Johnson and Cheng, 2001], it was deduced that (1) the transverse wave component at the magnetopause is not significantly amplified below a threshold magnetic shear angle (approximately 50 degrees), (2) greatest amplification is for magnetic shear between 70 and 180 degrees, and (3) waves with higher frequencies are less amplified.

Figure 2

Figure 2

\section{Kinetic Alfvén Waves at the Magnetopause}

These observations can be understood in the context of resonant mode conversion of compressional Alfvén waves into kinetic Alfvén waves (KAW) [Hasegawa, 1976] at the magnetopause. Resonant mode conversion occurs when a compressional Alfvén wave propagates into a region with gradients in $k_{\|} V_{A}$ such as at the magnetopause where the Alfvén velocity can increase by at least a factor of 10 . At the magnetopause, gradients in the direction normal to the magnetopause boundary are dominant compared with gradients along the boundary, and we can approximate the background plasma and magnetic field profiles as functions of the coordinate, $x$, in the direction normal to the magnetopause. We assume the magnetic field is of the form $\mathbf{B}=B_{0}(x) \mathbf{b}$ where $\mathbf{b}=\cos \theta_{b}(x) \hat{\mathbf{z}}+\sin \theta_{b}(x) \hat{\mathbf{y}}$ and the magnetic field angle, $\theta_{b}$ rotates by an angle $\theta_{s h}$ across the magnetopause. The equilibrium profiles vary smoothly across the magnetopause on a scale of 10 ion gyroradii $\left(\rho_{i}\right)$. For such a configuration, wave propagation is well-described by the kinetic-fluid model [Cheng and Johnson, 1999] which simplifies to the following set of dimensionless coupled equations for $\mathcal{W}_{\|}=\delta p+B_{0} \delta B_{\|}$ and $\mathcal{W}_{x}=i B_{0} \delta B_{x}$.

$$
\frac{d^{2} \mathcal{W}_{\|}}{d x^{2}}=-\left(k_{A}^{2}-k_{S}^{2}\right) \mathcal{W}_{\|}+\left(k_{A}^{2}-k_{\|}^{2}\right) \delta p
$$




$$
\begin{gathered}
+k_{\|}\left[\frac{d}{d x} \log \left(\frac{k_{\|}^{2}}{k_{A}^{2}}\right)\right] \mathcal{W}_{x} \\
\mathcal{K} \mathcal{W}_{x} \equiv\left[1+\frac{T_{e}}{T_{i}}\left(1+\hat{\eta} \frac{Z_{i}^{\prime}}{2}\right)\left(\frac{-2}{Z_{e}^{\prime}}\right)\right] k_{\|}^{2} \rho_{i}^{2} \frac{d^{2} \mathcal{W}_{x}}{d x^{2}} \\
=\left(k_{\|}^{2}-k_{A}^{2}\right) \mathcal{W}_{x}-k_{\|} \frac{d \mathcal{W}_{\|}}{d x} \\
\hat{\eta} \mathcal{W}_{x} \approx \int d x^{\prime} \frac{d k_{x}}{2 \pi} e^{i k_{x}\left(x-x^{\prime}\right)} b \frac{\left(\Gamma_{0}(b)-\Gamma_{1}(b)\right)}{\left(1-\Gamma_{0}(b)\right)} \mathcal{W}_{x}\left(x^{\prime}\right)
\end{gathered}
$$

$\mathbf{k}_{S}$ is the wavevector in the plane perpendicular to $x$; $k_{\|}=\mathbf{k} \cdot \mathbf{b}=\mathbf{k}_{S} \cdot \mathbf{b}=k_{S} \cos \theta_{s b}$, where $\theta_{s b}$ is the angle between $\mathbf{b}$ and $\mathbf{k}_{S}$; and $k_{A}^{2}=\omega^{2} / V_{A}^{2}$ is the Alfvén wavevector where $\omega$ is the wave frequency and $V_{A}$ is the Alfvén velocity. $Z_{s}^{\prime}$ is the derivative of the plasma dispersion function of argument $\zeta_{s}=\omega / \sqrt{2} k_{\|} v_{t s}$ for species $s$ with thermal velocity $v_{t s}$. We have taken the plasma to be isotropic. The operator $\hat{\eta}$ is a weakly nonlocal operator introduced by finite Larmor radius effects. The integration involves $\Gamma_{n}(b) \equiv I_{n}(b) e^{-b}$ where $b \equiv\left(k_{x}^{2}+k_{S}^{2} \sin ^{2} \theta_{b}\right) \rho_{i}^{2}$. Near the location where $k_{\|}^{2}=k_{A}^{2}, \hat{\eta} \approx 1+\mathcal{O}\left(\rho_{i}^{2} d^{2} / d x^{2}\right)$ and for $k_{A}^{2} \gg k_{\|}^{2}$ (as occurs when $k_{\|}$is small), the contribution of $\hat{\eta}$ vanishes.

The pressure equation required for the compressional wave is, $\delta p \approx(1-1 / \tau) \mathcal{W}_{\|}$where $\tau=1+\sum_{s} \beta_{s}\left(1+Z_{s}^{\prime} / 2\right)$ and summation is over all species, $s$. The function $1 / \tau$ is well behaved for the frequencies of interest (in contrast to the MHD approach which gives a singularity where $\left.\omega^{2}=k_{\|}^{2} C_{S}^{2} /(1+\beta)\right)$ [De Keyser et al., 1999]. In a cold, isotropic plasma $\zeta_{i} \gg 1, \tau \rightarrow 1$ and there is no contribution from this term. In a warm plasma with $\zeta \sim 1, \tau \sim \mathcal{O}(1)$ and this term only introduces weak damping to the compressional wave [Johnson and Cheng, 1997a], so the sound resonance is not very important. Moreover, near the Alfvén resonance where $k_{\|}^{2}=k_{A}^{2}$, the pressure term vanishes from Eq. 1, and Larmor radius corrections in the term proportional to $\delta p$ are not critical for describing wave behavior near the Alfvén resonance.

Generally, the Alfvén velocity increases across the magnetopause from the magnetosheath side so that $k_{A}^{2}$ is a monotonically decreasing function. In the magnetosheath the wave is propagating which requires $k_{A}^{2}>$ $k_{S}^{2}$. As the wave propagates across the magnetopause, $k_{A}$ decreases until $k_{A}^{2}=k_{S}^{2}$ where the compressional wave is cutoff. Beyond that location, the compressional wave decays. However, at the location $k_{A}^{2}=k_{\|}^{2}$ the decaying compressional wave encounters the Alfvén resonance where it can be reflected out of phase from the incoming wave. Near the resonance location the par- 
allel magnetic field is well behaved, but the transverse fields are singular.

Ion gyroradius effects resolve the singular behavior and are described by the kinetic response integral operator, $\mathcal{K}$ [Cheng and Johnson, 1999]. The model includes the effects of the parallel electric field through the quasineutrality condition, is valid for both large and small $k_{\perp}^{2} \rho_{i}^{2}$, and reduces to the KAW and inertial Alfvén wave dispersion relations in the appropriate limits. (Note that near the location where $k_{\|} \rightarrow 0$, the wave enters the inertial regime and decays on the scale of the electron skin depth [Cheng and Johnson, 1999]).

We solve Eqs. 1 and 2 numerically on a nonuniform discrete grid and obtain the solutions through matrix manipulation. Boundary conditions are imposed at the magnetosheath and magnetosphere boundaries. The boundary condition in the magnetosheath is an incoming compressional MHD wave. At the magnetosphere boundary, the compressional MHD wave is decaying. For the KAW only radiating/decaying solutions are allowed. Boundary conditions are imposed asymptotically. The Alfven velocity is taken to increase by a factor of 10 across the magnetopause and the magnetic field rotates through an angle, $\theta_{s h}$.

A good measure of the efficiency of mode conversion at the magnetopause is the amount of compressional wave absorption in the magnetopause layer. Energy absorption is determined by comparing the Poynting flux $(\delta \mathbf{E} \times \delta \mathbf{B} \cdot \hat{\mathbf{x}})$ of the incident compressional wave $\left(S_{I}\right)$ with the Poynting flux of the reflected $\left(S_{R}\right)$ and transmitted $\left(S_{T}\right)$ compressional waves. The Poynting flux of the KAW in the magnetopause near the mode conversion layer is $S_{K A W}=S_{I}+S_{R}-S_{T}$. In the magnetopause, the transverse magnetic field component is mainly from the KAW, therefore $S_{K A W} \sim$ $P_{\perp m p}$, and the compressional wave absorption, $A \equiv$ $\left(S_{I}+S_{R}-S_{T}\right) / S_{I}$ is proportional to the wave amplification, $P_{\perp m p} / P_{\perp m s h}$. Depending on the profiles of $V_{A}$ and $\mathbf{k}_{S} \cdot \mathbf{b}$, there can be up to three resonance locations in the magnetopause. The absorbed energy is converted to KAWs which: (a) propagate back into the magnetosheath (one resonance location), (b) propagate into both magnetosheath and magnetosphere (two resonance locations), or (c) couple to a quasi-trapped kinetic Alfvén wave (three resonance locations).

To determine the total absorption as a function of frequency and magnetic shear, we sum the absorption over the wavevector spectrum of incoming compressional waves. To do this, we assume that all wavevectors lie on a dispersion surface in wavevector space defined by $\omega(\mathbf{k})=$ const and integrate over the dispersion sur- 
face. We integrate over the angles of $\mathbf{k}: \theta_{k 0}$ is the angle between $k$ and the magnetic field in the magnetosheath and $\phi_{k 0}$ is the azimuthal angle in planes perpendicular to the magnetosheath magnetic field, $\mathbf{B}_{m s h}$. For compressional waves the dispersion surface is approximately defined by $\omega^{2} \approx k^{2}\left(V_{A}^{2}+C_{s}^{2} \sin ^{2} \theta_{k 0}\right)$. The wave vectors are approximately distributed on an ellipsoid with major radius $k=k_{A}$ and minor radii $k=k_{A} / \sqrt{1+C_{s}^{2} / V_{A}^{2}}$. The absorption spectrum as a function of frequency is obtained by integrating over the ellipsoid - that is, over the angles $\left(\theta_{k 0}, \phi_{k 0}\right)$ with $k^{2} \approx k_{A}^{2} /\left(1+C_{s}^{2} \sin ^{2} \theta_{k 0} / V_{A}^{2}\right)$ imposed by the dispersion relation. Moreover, compressional waves typically have $k_{\perp} \gg k_{\|}$so it is reasonable to assume that the spectrum is highly peaked around $\theta_{k 0}=\pi / 2$. On the other hand, there is no compelling reason to expect that the initial wave spectrum depends on the direction $\phi_{k 0}$.

The wave observations show a strong dependence of amplification $\left(P_{\perp m p} / P_{\perp m s h}\right)$ on the shear angle across the magnetopause. For small shear angles, there is little wave amplification, while above a threshold amplification is enhanced and relatively level. The minimum in amplification for small shear is consistent with the mode conversion mechanism because the waves in the magnetosheath propagate nearly perpendicular to the magnetic field. The absorption coefficient, $A\left(\omega, \theta_{s h}\right)$ is presented in Figure 3. The absorption is obtained by computing the absorption coefficient as a function of $\left(\omega, \theta_{s h}, \theta_{k 0}, \phi_{k 0}\right)$ and performing an integration over the variables $\left(\theta_{k 0}, \phi_{k 0}\right)$ with uniform weight in $\phi_{k 0}$ and a strongly peaked weighting function about $\theta_{k 0}=\pi / 2$.

The absorption is the result of mode conversion to KAWs and measures the efficiency of the mode conversion mechanism. The absorbed energy is the Poynting flux of the KAW which radiates away from the mode conversion location. The Poynting fluxes scale as the group velocity multiplied by spectral density. Because the KAW radiates slowly across the magnetic field, its amplitude must be greatly increased compared with the amplitude of the incoming MHD wave in order to carry away the mode converted energy from the field line resonance location. For KAWs the Poynting flux is approximately, $S_{K A W} \sim\left(\omega / k_{x}\right) k_{x}^{2} \rho^{2} /\left(1+k_{x}^{2} \rho^{2}\right) P_{\perp}$, while for the MHD wave $S_{M H D} \sim V_{A} P_{\perp}$. The KAW wavevector, $k_{x}$ can be estimated from dominant balance in Eq. $2-k_{x} \sim\left(\rho_{i}^{2} L\right)^{-1 / 3}$ where $L$ is the scale length of the Alfvén velocity gradient at the magnetopause. One can then estimate from the linear dispersion relation that the wave amplification $P_{\perp K A W} / P_{\perp M H D} \sim$ $A\left(1+(\rho / L)^{2 / 3}\right) V_{A} /\left[2 \pi f \rho(\rho / L)^{1 / 3}\right]$. For typical magnetopause parameters: $V_{A} \sim 300 \mathrm{~km} / \mathrm{s}, \rho_{i} \sim 50 \mathrm{~km}$, 
$L \sim 500 \mathrm{~km}, f=25 \mathrm{mHz}, P_{\perp K A W} / P_{\perp M H D} \sim 100 A$.

Because $\left|\delta \mathbf{B}_{\perp}^{2}\right|$ amplification scales directly with compressional wave absorption, the results of Figure 3 can be compared qualitatively with observed $\left|\delta \mathbf{B}_{\perp}^{2}\right|$ amplification. The important features to notice are: (1) for angles greater than $50^{\circ}$ the absorption is approximately constant, but for smaller shear there is a trough in $A$ and (2) the absorption decreases weakly as frequency increases for angles larger than $50^{\circ}$. However, for angles less than $50^{\circ}$ there is a significant broadening of the trough for higher frequency with far less absorption. These qualitative properties correspond well to observations of $\left|\delta \mathbf{B}_{\perp}^{2}\right|$ amplification as a function of magnetic shear angle and frequency as discussed in Fig. 2. The quantitative differences between the theory and data (for example, the theoretical threshold angle is smaller) can be attributed to the uncertainly involved in analyzing the data and the simplifications of the theoretical model.

Figure 3

\section{Plasma Heating and Particle Transport} Associated with Kinetic Alfvén Waves

Large amplitude transverse wave activity accompanies nearly every magnetopause crossing [Perraut et al., 1979; Rezeau et al., 1993; Song et al., 1993c; Song, 1994; Phan and Paschmann, 1996; Johnson et al., 2001].Often the observed wave amplitudes at the magnetopause can be significantly large compared with the background magnetic field, $B_{0}$. It is not uncommon for $\delta B_{\perp} / B_{0}$ to be the order of 0.2 or even larger [Rezeau et al., 1993; Song et al., 1993c, 1993b]. Moreover, in the cusp, it is likely that $\delta B_{\perp} / B_{0} \sim 1$ because of the weak background field.

The magnetopause provides a boundary between magnetosheath plasma and magnetospheric plasma. Plasma that leaks into the boundary layer is often found to have distinctive particle distributions indicative of acceleration processes. For example, electron distributions in the boundary layer are often found to be accelerated in the direction parallel to the magnetic field. These distributions have been identified as signatures of reconnection events, but the process which accelerates the electrons is not well known. It was suggested that electrons could also be accelerated by kinetic Alfvén waves [Hasegawa and Chen, 1976; Lee et al., 1994] which preferentially heat electrons in the parallel direction due to the parallel electric field. Thermal electrons trapped in the wave potential would be heated leading to a slight increase in the parallel direction consistent with observations. 
On the other hand, ions in the sheath transition layer and boundary layers often exhibit significant anisotropy with $T_{\perp}>T_{\|}$[Anderson et al., 1991; Song et al., 1993a]. Moreover, Wilber et al. [2001] have reported unusual low energy ion distribution components in the low-latitude boundary layer with pitch angles intermediate between 0 and 90 degrees observed by WIND/3DP during equatorial passes. The particles appeared to have undergone adiabatic streaming from a stronger magnetic field region with heating occuring near the magnetopause as deduced from the mirror ratios. The low energy ions appear to have been heated perpendicular to the magnetic field and in some events the core of the distribution appears to be flattened. The ion distributions presented by Song et al. [1993a] also illustrate that in the inner boundary layer the slope of the low energy component of the ion distribution function is flattened compared with the magnetosheath distribution suggestive that a physical process may be heating the low energy core of the distribution to higher energies.

In a recent study Johnson and Cheng [1997b] showed that when the kinetic Alfvén wave amplitude is sufficiently large, particle orbits can become stochastic leading to significant transport and particle heating. When the orbits became stochastic in an inhomogeneous background field, the particles could diffuse rapidly across the magentic field with a diffusion coefficient of $D \sim$ $10^{9} \mathrm{~m}^{2} / \mathrm{s}$.

In this paper we will concentrate on particle heating. To study ion heating in the presence of a kinetic Alfvén wave, we will prescribe the electromagnetic fields consistent with the kinetic Alfvén waves. We investigate the particle motion in those prescribed fields as a function of the wave amplitude. The study will consist of a sequence of Poincaré sections taken at different wave amplitude which demonstrate the onset of stochasticity. The results will demonstrate: (1) stochastic ion heating can result through nonlinear coupling between low frequency waves and cyclotron motion, (2) ions can be heated transverse to the magnetic field leading to temperature anisotropy $\left(T_{\perp}>T_{\|}\right)$as observed at the magnetopause [Wilber et al., 2001], and (3) the stochastic process will deplete the core of the ion distribution function leading to a flattened core of the distribution function similar to the observations of [Wilber et al., 2001; Song et al., 1993a].

\section{Kinetic Alfvén Waves and Ion Heating}

The kinetic Alfvén wave [Hasegawa, 1976] is well described by three scalar quantities- $\phi, A_{\|}$, and $\delta B_{\|}$ [Cheng and Johnson, 1999]. The fields associated with 
the kinetic Alfvén wave are obtained through Maxwell's equations given in Gaussian units.

$$
\begin{gathered}
\mathbf{E}=-\nabla \phi-\frac{1}{c} \frac{\partial A_{\|} \mathbf{b}}{\partial t} \\
\mathbf{B}=\mathbf{B}_{\mathbf{0}}(\mathbf{x})+\delta \mathbf{B} \approx\left(B_{0}(x)+\delta B_{\|}\right) \mathbf{b}-\mathbf{b} \times \nabla A_{\|}
\end{gathered}
$$

where $\mathbf{b}$ is the unit vector in the direction of the magnetic field. The vector potential is related to the electrostatic potential by introduction of a secondary potential, $\psi$ defined by

$$
E_{\|}=-\nabla \psi=-\nabla_{\|} \phi-\frac{1}{c} \frac{\partial A_{\|}}{\partial t}
$$

The fields are obtained by solving the set of equations described in Cheng and Johnson [1999] for the prescribed background magnetic field. If the background field is uniform, the kinetic Alfvén wave is a simple sinusoidal with $\phi=\phi_{0} \cos (\mathbf{k} \cdot \mathbf{x}-\omega t), \psi \approx$ $-\left(T_{e} / T_{i}\right) k_{\perp}^{2} \rho_{i}^{2} \phi /\left(1+k_{\perp}^{2} \rho_{i}^{2}\right), A_{\|}=\left(k_{\|} / \omega\right)(\phi-\psi)$. The wave satisfies the approximate dispersion relation

$$
\omega^{2}=k_{\|}^{2} V_{A}^{2}\left(1+\left(1+\frac{T_{e}}{T_{i}}\right) k_{\perp}^{2} \rho_{i}^{2}\right)
$$

where we ignore damping and take a Padé approximation for the Bessel function as described in Cheng and Johnson [1999]. The kinetic Alfvén wave is incompressible at low $\beta$ so that $\delta B_{\|} \approx 0$, but at $\beta \sim 1$ and short wavelength, $\delta B_{\|}$may also included as prescribed in Cheng and Johnson [1999]. For the chosen parameters $\left(k_{\perp} \rho_{i}=3, k_{\|} \rho_{i}=0.05\right.$, and $\left.\beta=1\right),\left|\delta B_{\|}\right| \sim 0.55\left|\delta B_{\perp}\right|$ and is $90^{\circ}$ out of phase.

Particle orbits are determined by the equation of motion

$$
m_{i} \frac{d^{2} \mathbf{r}}{d t}=q_{i}\left(\mathbf{E}+\frac{\mathbf{v}}{c} \times \mathbf{B}\right)
$$

which we normalize to

$$
\ddot{\mathbf{X}}=-\tilde{\nabla} \Phi+\dot{\mathbf{X}} \times\left(\Delta \hat{\mathbf{b}}+\frac{\delta \mathbf{B}}{\tilde{B}_{0}}\right)
$$

where $\mathbf{X}=\mathbf{r} / \rho_{i}, \tau=\Omega t, \tilde{\nabla}=\rho_{i} \nabla, \Phi=q_{i} \phi / T_{i}$, with $\Omega=q_{i} \tilde{B}_{0} / m_{i} c, \rho_{i}=\sqrt{T_{i} / m_{i}} / \Omega, \Delta=\left|B_{0}(x) / \tilde{B}_{0}\right|$ and $\dot{\mathbf{X}} \equiv d \mathbf{X} / d \tau$, and $\tilde{B}_{0}$ is a characteristic value of magnetic field.

To investigate the behavior of particles in the kinetic Alfvén waves, we plot Poincaré sections for particle orbits. This technique is standard and has been applied to electrostatic waves to understand plasma heating well above the cyclotron frequency [Karney and Bers, 1977], at near the cyclotron frequency [Hsu et al., 
1979]. Points on Poincaré sections are plotted at constant particle gyrophase, $\gamma$, with the requirement that $\dot{\gamma}<0$. In the absence of waves, this would correspond with one point per gyroperiod. At each crossing of the phase space plane defined by $\gamma=0$ where $\mathbf{k} \cdot \mathbf{b} \times \dot{\mathbf{X}}=k_{\perp} v_{\perp} \sin (\gamma)$, we plot the value of the magnetic moment, $\mu \equiv|\mathbf{b} \times \dot{\mathbf{X}}|^{2} / \Delta$, versus $\Psi=\mathbf{k} \cdot \mathbf{x}-\omega t$, taken modulo $2 \pi$. In the following plots, at least 1000 points are taken for each trajectory to resolve the phase space structure (more near a separatrix). The choice of these variables is good for examining particle heating in the presence of the wave because the magnetic moment is an adiabatic invariant which follows well defined trajectories in phase space. The wave phase is an obvious choice because there is a direct correlation between the adiabatic particle motion and the wave amplitude.

To examine the onset of stochastic particle behavior in the presence of large amplitude kinetic Alfvén waves, we examine a sequence of Poincaré sections as a function of wave magnetic field amplitude. For simplicity, we assume a uniform background magnetic field. We specify $k_{\perp} \rho_{i}=3, \omega=\Omega_{i} / 5, \beta=1, T_{e} / T_{i}=0.2$ and $k_{\|} \rho_{i} \approx 0.05$ consistent with the kinetic Alfvén wave dispersion relation. The small perpendicular scales are consistent with typical kinetic Alfvén wave solutions at the magnetopause with wavelength the order of $100 \mathrm{~km} \approx 2 \rho_{i}$ [Johnson and Cheng, 1997b; Johnson et al., 2001].

For clarity of Poincaré section plots, we take the wave frequency equal to $1 / 5$ of the ion gyrofrequency. For smaller frequency similar physics leads to stochastic thresholds, but the formation of island chains composed of hundreds of islands cannot be as easily seen by eye. Interestingly, the stochastic threshold primarily depends on wave amplitude and is not strongly dependent on wave frequency in the range of interest. Note that because $k_{\perp} \rho_{i}$ is larger than 1 , the kinetic Alfvén wave does have a significant electrostatic component associated with ion Larmor radius effects.

The Poincaré section for $\delta B_{\perp} / B_{0}=0.006$ is shown in Figure 4. Particles are started with $\Psi=0$ with varying initial value of $\mu$ and $v_{\|}=0$. If there were no wave, the particles would simply gyrate with constant value of $\mu$. For small wave amplitude, $\mathbf{X}$ is nearly periodic in the gyrophase, so dependence of $\Psi$ on time is primarily through $-\omega t$. Because the wave frequency is $\Omega_{i} / 5, \Psi$ will approximately decrease by $2 \pi / 5$ each gyroperiod until it returns to the original phase (minus $2 \pi)$. The Poincare section for a given particle would therefore reduce to five equally spaced points at constant $\mu$. However, with the addition of the wave, the particle gyration can be retarded or accelerated. 
Figure 4

In Figure 4, it is apparent that the for initial $\mu<0.41$ particles do not return to the same wave phase after a wave period (five gyroperiods), but have a small positive increment in phase $\Psi$. On the other hand, for initial $\mu>0.41$, it is apparent that the after a wave period, the phase, $\Psi$, of the particle will have a small negative increment. Near initial $\mu=0.41$, it is evident that there is a boundary where the orbit is stationary and the Poincaré section only consists of only five points. As the amplitude is increased these fixed points move to larger values of $\mu$ and nearby orbits circulate around the fixed points. Moreover, other boundaries appear across which the phase advances/retards, but with higher order periodicity. This phenomenon is illustrated in Figure 5 which shows the Poincaré section for $\delta B_{\perp} / B_{0}=0.05$. The period five island chain associated with the original transition boundary has moved to larger value of $\mu$ and is quite large in extent. Above that chain, the high energy particles do not show any structure related to the gyromotion and simply float up and down in the wave. Other islands chains have also appeared at lower energies. The obvious island periods evidently are in the sequence: $26,21,58,16,27$ and so forth. Islands in the period 16 and 21 chains have just begun to overlap.

Figure 5

At the lowest energies, a chain of five fingers (rather than islands) has appeared. The fingers have divided into ten subfingers. The central finger contains two islands.. The physical origin of the fingers is the increase of the $\mathbf{E} \times \mathbf{B}$ velocity of the wave. When low energy particles are subjected to large $\mathbf{E} \times \mathbf{B}$ motion, the gyrophase can reverse direction and the particles are trapped in the wave. As a result, the particles are not sampled during their gyromotion and the island is incomplete (that is, the low energy particles can skip one or more fingers during their gyromotion).

Figure 6

A slight increase in wave amplitude shown in Figure 6 allows the phase space islands to merge and regions of stochastic orbits appear. Island structures still remain embedded in the stochastic region, but now particle trajectories can wander through the stochastic sea to higher energies than previously accessible. Hence, the low energy part of the ion distribution can be effectively heated. Note that the ten finger structures have now moved into the stochastic sea and comprise a sequence of ten islands. A new five fingered structure has also begun to emerge from the low energy part of the phase space with regular orbits below the stochastic sea with a fixed point in the central island. The stationary
Figure 4

Figure 5

Figure 6 
orbit at $\Psi=0$ in the central finger is an elongated orbit which has period equal to that of the wave and can be considered to be in nonlinear resonance with the wave. Above the stochastic sea, particle trajectories lie on well defined curves or island chains and the particles are not heated. The large period five island chain remains intact and moves to higher energy. However, clear boundaries still confine heating to the lowest energy ions.

For $\delta B_{\perp} / B_{0} \sim 0.11$ as shown in Figure 7 nearly the entire low $\mu$ region becomes stochastic except for a few small islands that remain embedded in the stochastic sea. The stochastic region is forced up against the set of period five islands which have themselves become stochastic. A clear boundary exists between the two stochastic regions and particles may not move across that boundary. Above the boundary, the period five island chain has also merged and become stochastic and other chains of islands have also appeared against the boundary between the lower stochastic region and the upper stochastic region. Chains of islands have also appeared inside the period five islands. However, the two regions are still separated and low energy particles cannot be energized much above the thermal speed. With a modest increase to $\delta B_{\perp} / B_{0}=0.14$ as shown in Figure 8, the period five islands merge with the low energy stochastic region leaving a path for low energy particles to be heated well beyond the thermal energy. With further increase in the wave amplitude the stochastic regime pushes to larger $\mu$. For example, with $\delta B_{\perp} / B_{0} \sim 0.3$ particles are readily energized to $\mu \sim 4$.

Figure 7

Figure 8

These results have several implications relevant to Figure 7 magnetopause observations. First, transverse ion heating due to this process depends on wave amplitude. Below the wave threshold, there is no heating of the plasma. Slightly above the threshold, the core of the distribution is expected to flatten, but ions are not heated above the thermal velocity. For larger wave amplitude, ions can be heated above the thermal velocity. The heating process can occur rapidly over a time less than 30 cyclotron periods. Due to the brevity of this letter, we defer estimates of the stochastic threshold, more detailed description of the appearance of island chains, island overlap,, and discussion of the effect of background gradients (and the resulting particle transport) for a later publication.

Obviously, wave heating at the magnetopause is more complicated than this simple picture. This calculation is primarily meant to provide understanding of the non- 
linear coupling between kinetic Alfvén waves and cyclotron motion and to provide a physical picture which gives qualitative understanding of resulting particle signatures. Most likely, there is a spectrum of waves that can participate in the heating process. Typically, the addition of a second wave or magnetic field rotation can reduce the threshold for stochasticity to occur and increase particle heating and transport beyond quasilinear levels [Johnson and Cheng, 1997c].

\section{SUMMARY}

In this paper we have examined the role of kinetic Alfvén waves at the magnetopause. ULF waves dominate the spectrum of nearly every magnetopause crossing. Probably the most distinctive wave features at the magnetopause are the large increase in wave amplitude and abrupt change in wave polarization where the magnetopause Alfvén velocity gradient is encountered. The abrupt change in wave characteristics is suggestive of a process which could convert the compressional wave into a shear wave. Theoretical examination of the mode conversion process yields results similar to the observations. Compressional wave mode convert into kinetic Alfvén waves at the magnetopause. The mode structure suggests that wave amplitude will be increased and polarization will change abruptly. Magnetic rotation across the magnetopause provides a good test of the theory of resonant mode conversion. Wave observations appear to be in rough agreement with the theory which predicts small wave amplification below a threshold shear angle and roughly constant amplification above the shear angle. The amplification as a function of frequency also seems to agree qualitatively with observations. It therefore seems reasonable to believe that the ULF wave activity at the magnetopause is the result of a mode conversion process.

That a population of large amplitude ULF waves nearly always populates the magnetopause and the associated boundary layers is not without consequence. Quasilinear theory predicts substantial plasma transport for typical observed parameters. Moreover, because the waves have such large amplitude, the orbits can also be stochastic leading to rapid transport and heating. We examined single particle orbits in kinetic Alfvén waves and demonstrated that ions can be significantly heated for modest wave amplitudes. Recent particle observations near the magnetopause and in the low-latitude boundary layer show significant heating of low energy ions without significant heating of the energetic population. Such observations are a typical signature of stochastic ion heating near the stochastic thresh- 
old. Moreover, in plasma with magnetic shear, stochastic ion transport can even exceed the quasilinear particle entry estimate of $10^{27}$ particles/sec. Hence, kinetic Alfvén waves found at the magnetopause contribute to the population of the boundary layers and can explain some of the observed wave and particle signatures found in those layers.

Acknowledgments. This work is supported by the NSF grant ATM-9906142 and DoE Contract CGLNo. DE-AC0276-CHO3073.

\section{REFERENCES}

Anderson, B. J., S. A. Fuselier, and D. Murr, Electromagnetic ion cyclotron waves observed in the plasma depletion layer, Geophys. Res. Lett., 18, 1955, 1991.

Belmont, G., F. Reberac, and L. Rezeau, Resonant amplification of magnetosheath MHD fluctuations at the magnetopause, Geophys. Res. Lett., 22, 295-298, 1995.

Cheng, C. Z., and J. R. Johnson, A kinetic-fluid model, J. Geophys. Res., 104, 413-427, 1999.

De Keyser, J., M. Roth, F. Reberac, L. Rezeau, and G. Belmont, Resonant amplification of MHD waves in realistic subsolar magnetopause configurations, J. Geophys. Res., 104, 2399-2409, 1999.

Fuselier, S. A., The LLBL for northward IMF: Open or closed, AGU Chapman Conference, The Low Latitude Boundary Layer, New Orleans, LA, 2001.

Hasegawa, A., Particle acceleration by MHD surface wave and formation of aurora, J. Geophys. Res., 81, 5083-5090, 1976.

Hasegawa, A., and L. Chen, Parametric decay of kinetic Alfvén wave and its application to plasma heating, Phys. Rev. Lett., 36, 1362-1365, 1976.

Hsu, J. Y., K. Matsuda, M. S. Chu, and T. H. Jensen, Stochastic heating of a large-amplitude standing wave, Phys. Rev. Lett., 43, 203-206, 1979.

Johnson, J. R., and C. Z. Cheng, Global structure of mirror modes in the magnetosheath, J. Geophys. Res., 102, 71797189, 1997a.

Johnson, J. R., and C. Z. Cheng, Kinetic Alfvén waves and plasma transport at the magnetopause, Geophys. Res. Lett., 24, 1423-1426, 1997b.

Johnson, J. R., and C. Z. Cheng, Plasma transport at the magnetopause due to low frequency mhd waves in a strongly sheared magnetic field, Eos Trans. AGU, 78(46), F591, Fall Meet. Suppl., 1997c.

Johnson, J. R., and C. Z. Cheng, Signatures of mode conversion and kinetic Alfvén waves at the magnetopause, Geophys. Res. Lett., 28, 227-230, 2001.

Johnson, J. R., C. Z. Cheng, and P. Song, Signatures of mode conversion and kinetic Alfvén waves at the magnetopause, Geophys. Res. Lett., 28(2), 227-230, 2001.

Karney, C. F. F., and A. Bers, Stochastic ion heating by a perpendicularly propagating electrostatic wave, Phys. Rev. Lett., 39, 550, 1977.

Lee, L. C., J. R. Johnson, and Z. W. Ma, Kinetic Alfvén waves as a source of plasma transport at the dayside magnetopause, J. Geophys. Res., 99, 17405-17411, 1994. 
Perraut, S., R. Gendrin, P. Robert, and A. Roux, Magnetic pulsations observed onboard GEOS 2 in the ULF ran ge during multiple magnetopause crossings, in Eur. Space Agency Spec. Publ., 148, pp. 113-122. 1979.

Phan, T., Formation of the LLBL by merging, AGU Chapman Conference, The Low Latitude Boundary Layer, New Orleans, LA, 2001.

Phan, T. D., and G. Paschmann, Low-latitude dayside magnetopause and boundary layer for high magnetic shear 1 . structure and motion, J. Geophys. Res., 101, 1996.

Phan, T. D., et al., Low-latitude dusk flank magnetosheath, magnetopause, and boundary layer for low magnetic shear: Wind observations, J. Geophys. Res., 102, 1997.

Rezeau, L., A. Roux, and C. T. Russell, Characterization of small-scale structures at the magnetopause from ISEE measurements, J. Geophys. Res., 98, 179, 1993.

Song, P., Observations of waves at the dayside magnetopause, in Solar Wind Source of Magnetospheric UltraLow-Frequency Waves, Geophysical Monograph Series, vol. 81, pp. 159-171. 1994.

Song, P., et al., Wave properties near the subsolar magnetopause for northward interplanetary magnetic field: Multiple instrument particle observations, J. Geophys. Res., 98, 11,319-11,337, 1993a.

Song, P., C. T. Russell, and C. Y. Huang, Wave properties near the subsolar magnetopause: Pc 1 waves in the sheath transition layer, J. Geophys. Res., 98, 5907-5923, 1993b.

Song, P., C. T. Russell, R. J. Strangeway, J. R. Wygant, C. A. Cattell, R. J. Fitzenreiter, and R. R. Anderson, Wave properties near the subsolar magnetopause: Pc 34 energy coupling for northward interplanetary magnetic field, J. Geophys. Res., 98, 187-196, 1993c.

Wilber, M., G. K. Parks, and R. P. Lin, Conic-like ion components observed interior to the dusk magnetopause, AGU Chapman Conference, The Low Latitude Boundary Layer, New Orleans, LA, 2001.

Jay R. Johnson and C. Z. Cheng, Princeton University, Plasma Physics Laboratory, Princeton, NJ 08543. (e-mail jrj@pppl.gov, fcheng@pppl.gov) 


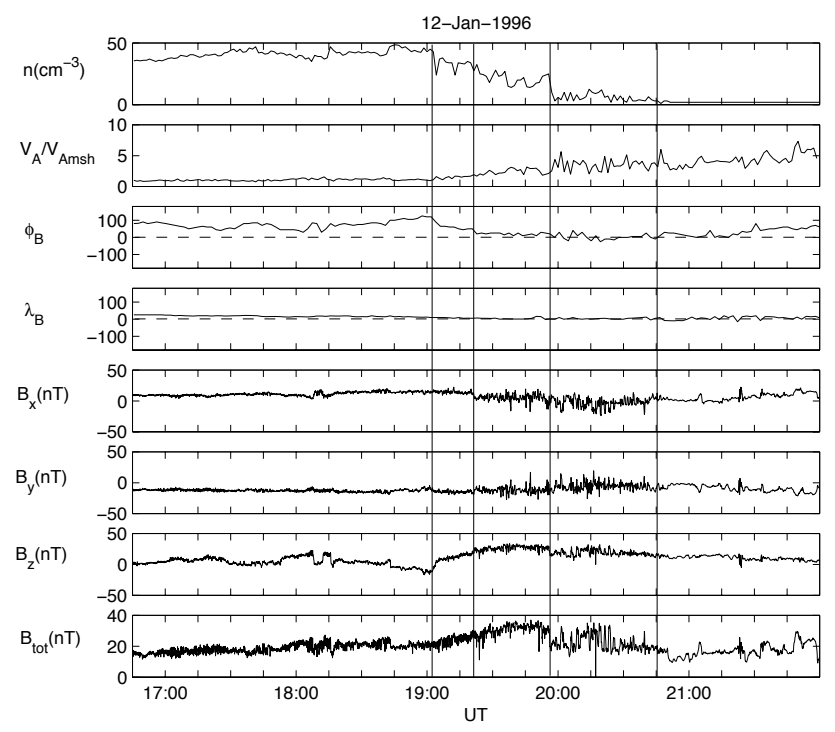

Figure 1. Density, Alfvén velocity, and magnetic field data from a magnetopause crossing by the WIND satellite on Jan 12, 1996 from approximately 19:20 UT to 19:55 UT. $\phi_{B}$ is the magnetic field vector angle in the LM plane (zero along $\mathrm{L}$ axis) and the $\lambda_{B}$ is the angle between the magnetic field and the magnetopause tangent plane for a LMN boundary normal coordinate system. Note that in the magnetosheath (prior to 19:20 UT) wave power is primarily compressional. Coincident with the density gradients at the magnetopause is a strong enhancement in the transverse components of the magnetic field fluctuations, but the compressional amplitude remains approximately the same as in the magnetosheath. Data courtesy of R. Lepping and Phan et al., [1997].

Figure 1. Density, Alfvén velocity, and magnetic field data from a magnetopause crossing by the WIND satellite on Jan 12, 1996 from approximately 19:20 UT to 19:55 UT. $\phi_{B}$ is the magnetic field vector angle in the LM plane (zero along L axis) and the $\lambda_{B}$ is the angle between the magnetic field and the magnetopause tangent plane for a LMN boundary normal coordinate system. Note that in the magnetosheath (prior to 19:20 UT) wave power is primarily compressional. Coincident with the density gradients at the magnetopause is a strong enhancement in the transverse components of the magnetic field fluctuations, but the compressional amplitude remains approximately the same as in the magnetosheath. Data courtesy of R. Lepping and Phan et al., [1997]. 


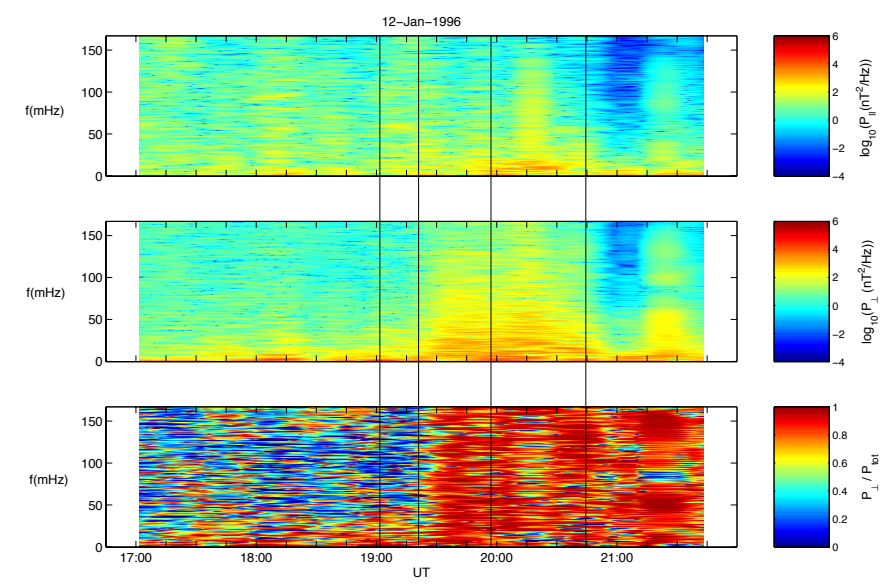

Plate 1. Wave power spectra for the WIND crossing of the magnetopause shown in Fig. 1. $P_{\|}, P_{\perp}$ and $P_{\text {tot }}$ are the power spectral densities obtained from $\left|\delta B_{\|}\right|^{2},\left|\delta B_{\perp}\right|^{2}$ and $|\delta \mathbf{B}|^{2}$ respectively. Notice that prior to crossing the magnetopause wave power is primarily compressional, coincident with the magnetopause crossing (indicated by the vertical line), the wave activity is primarily transverse. Note that the compressional wave component remains essentially the same before and during the magnetopause crossing.

Plate 1. Wave power spectra for the WIND crossing of the magnetopause shown in Fig. 1. $P_{\|}, P_{\perp}$ and $P_{t o t}$ are the power spectral densities obtained from $\left|\delta B_{\|}\right|^{2},\left|\delta B_{\perp}\right|^{2}$ and $|\delta \mathbf{B}|^{2}$ respectively. Notice that prior to crossing the magnetopause wave power is primarily compressional, coincident with the magnetopause crossing (indicated by the vertical line), the wave activity is primarily transverse. Note that the compressional wave component remains essentially the same before and during the magnetopause crossing. 


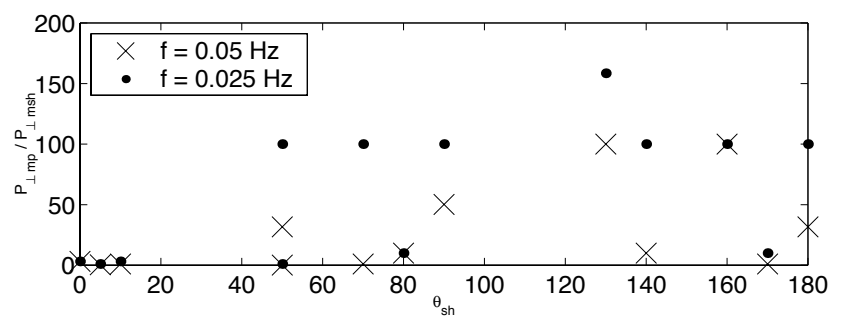

Figure 2. Amplification $\left(P_{\perp m p} / P_{\perp m s h}\right)$ of waves at the magnetopause as a function of magnetic shear.

Figure 2. Amplification $\left(P_{\perp m p} / P_{\perp m s h}\right)$ of waves at the magnetopause as a function of magnetic shear. 


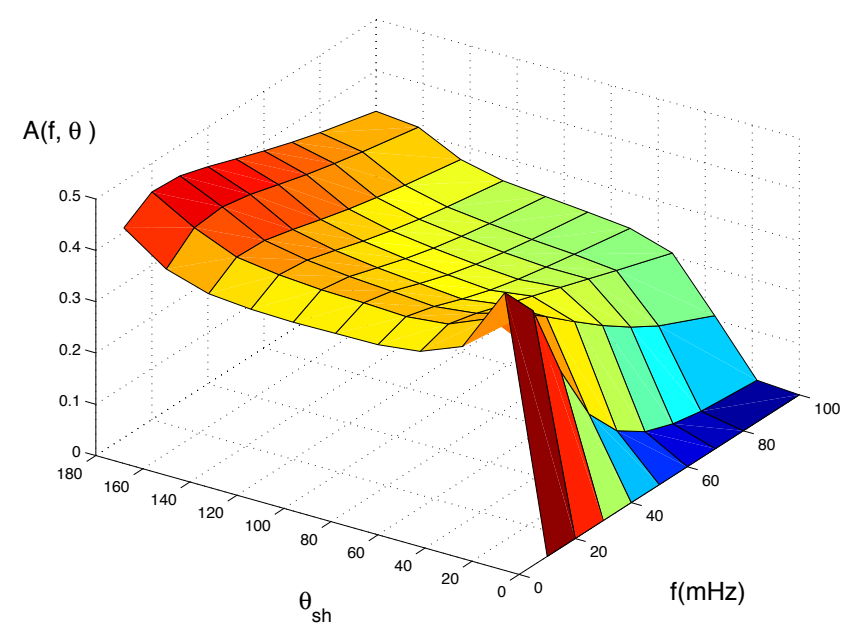

Figure 3. Absorption coefficient as a function of frequency and magnetic shear angle $\theta_{s h}$ (degrees).

Figure 3. Absorption coefficient as a function of frequency and magnetic shear angle $\theta_{s h}$ (degrees).

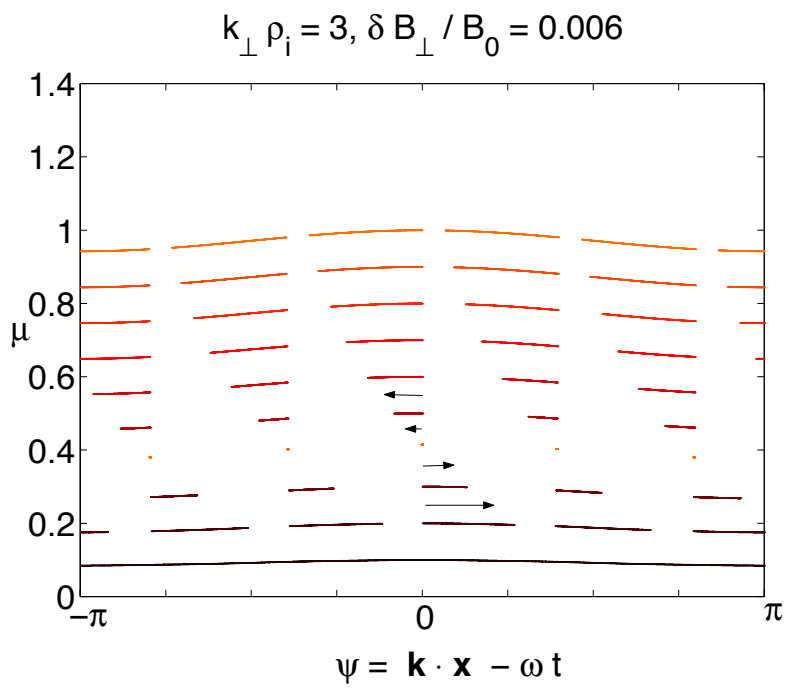

Figure 4. Poincaré section for small amplitude wave. Note that low energy particles are advanced in the phase of the wave while higher energy particles are retarded. Phase space islands with period five first emerge along the stationary phase trajectory.

Figure 4. Poincaré section for small amplitude wave. Note that low energy particles are advanced in the phase of the wave while higher energy particles are retarded. Phase space islands with period five first emerge along the stationary phase trajectory. 


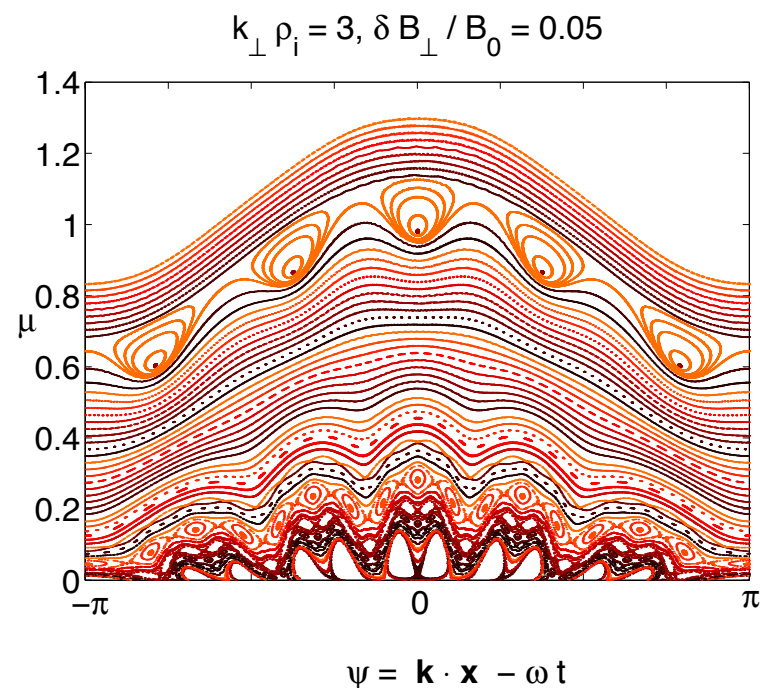

Figure 5. As the wave amplitude is increased, chains of phase space islands appear. The period 16 island chain has just begun to merge and a separatrix appears.

Figure 5. As the wave amplitude is increased, chains of phase space islands appear. The period 16 island chain has just begun to merge and a separatrix appears. 


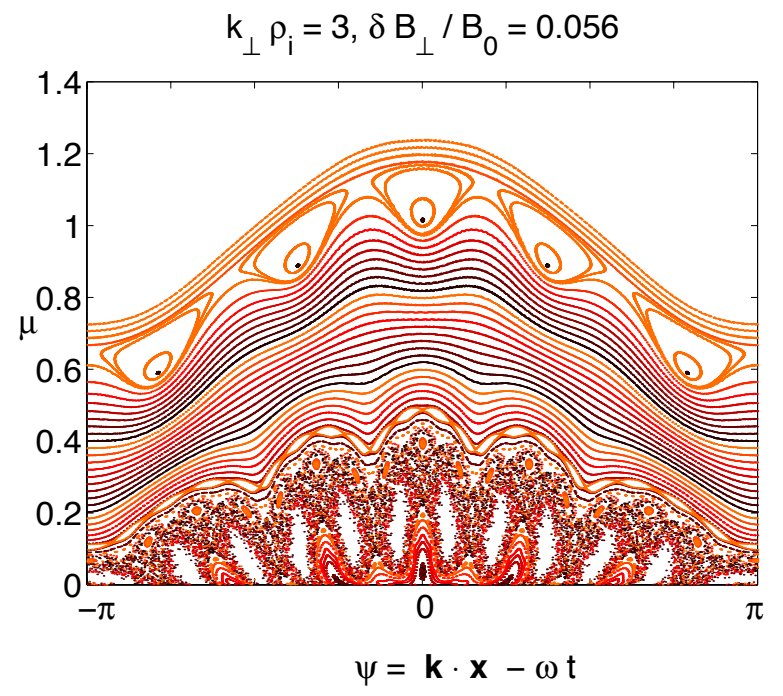

Figure 6. With a slight increase in wave amplitude, the low energy region of the Poincaré section becomes stochastic. Low energy particles can now wander through the stochastic sea to higher energies. Phase space islands remain embedded in the stochastic sea, but the entire region becomes stochastic for $\delta B / B_{0}=0.056$.

Figure 6. With a slight increase in wave amplitude, the low energy region of the Poincaré section becomes stochastic. Low energy particles can now wander through the stochastic sea to higher energies. Phase space islands remain embedded in the stochastic sea, but the entire region becomes stochastic for $\delta B / B_{0}=0.056$. 


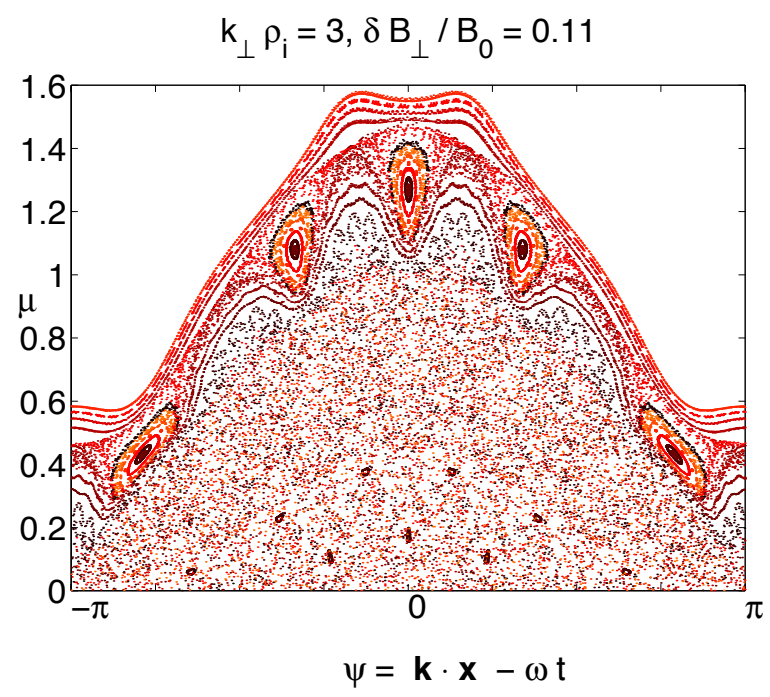

Figure 7. Both the period five island chain and the low energy region are stochastic, but they are separated by a boundary. Global stochasticity occurs when the two regions merge just above $\delta B_{\perp} / B_{0}=0.11$.

Figure 7. Both the period five island chain and the low energy region are stochastic, but they are separated by a boundary. Global stochasticity occurs when the two regions merge just above $\delta B_{\perp} / B_{0}=$ 0.11 . 


$$
\mathrm{k}_{\perp} \rho_{\mathrm{i}}=3, \delta \mathrm{B}_{\perp} / \mathrm{B}_{0}=0.14
$$

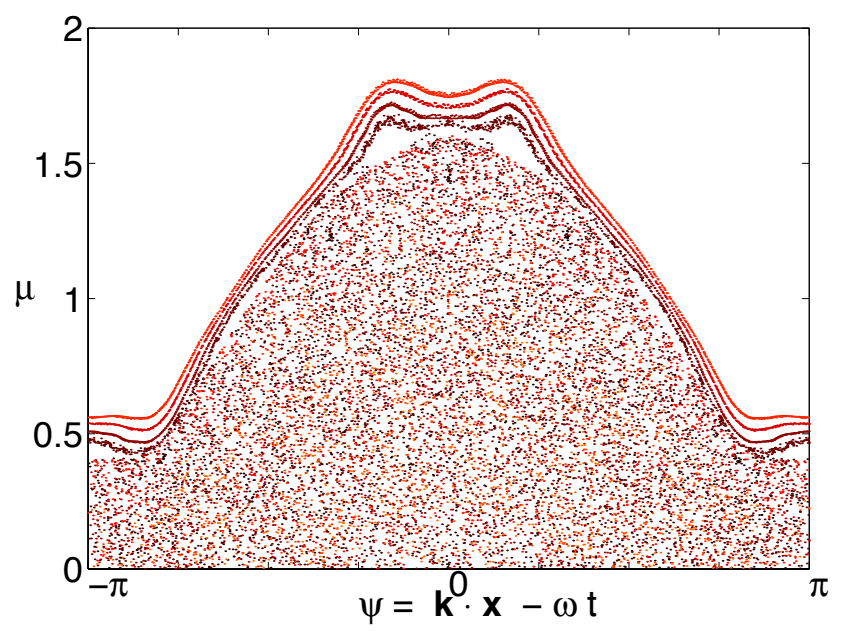

Figure 8. Global stochasticity for $\delta B_{\perp} / B_{0}=0.14$. Cold and/or thermal particles may wander to superthermal energies resulting in heating perpendicular to the magnetic field.

Figure 8. Global stochasticity for $\delta B_{\perp} / B_{0}=0.14$. Cold and/or thermal particles may wander to superthermal energies resulting in heating perpendicular to the magnetic field. . 


\section{External Distribution}

Plasma Research Laboratory, Australian National University, Australia

Professor I.R. J ones, Flinders University, Australia

Professor J oão Canalle, Instituto de Fisica DEQ/IF - UERJ , Brazil

Mr. Gerson O. Ludwig, Instituto Nacional de Pesquisas, Brazil

Dr. P.H. Sakanaka, Instituto Fisica, Brazil

The Librarian, Culham Laboratory, England

Library, R61, Rutherford Appleton Laboratory, England

Mrs. S.A. Hutchinson, JET Library, England

Professor M.N. Bussac, Ecole Polytechnique, France

Librarian, Max-Planck-Institut für Plasmaphysik, Germany

J olan Moldvai, Reports Library, MTA KFKI-ATKI, Hungary

Dr. P. Kaw, Institute for Plasma Research, India

Ms. P.J . Pathak, Librarian, Insitute for Plasma Research, India

Ms. Clelia De Palo, Associazione EURATOM-ENEA, I taly

Dr. G. Grosso, Instituto di Fisica del Plasma, Italy

Librarian, Naka Fusion Research Establishment, J AERI, J apan

Library, Plasma Physics Laboratory, Kyoto University, J apan

Research Information Center, National Institute for Fusion Science, J apan

Dr. O. Mitarai, Kyushu Tokai University, J apan

Library, Academia Sinica, Institute of Plasma Physics, People's Republic of China

Shih-Tung Tsai, Institute of Physics, Chinese Academy of Sciences, People's Republic of China

Dr. S. Mirnov, TRINITI, Troitsk, Russian Federation, Russia

Dr. V.S. Strelkov, Kurchatov Institute, Russian Federation, Russia

Professor Peter Lukac, Katedra Fyziky Plazmy MFF UK, Mlynska dolina F-2, Komenskeho Univerzita, SK-842 15 Bratislava, Slovakia

Dr. G.S. Lee, Korea Basic Science Institute, South Korea

Mr. Dennis Bruggink, Fusion Library, University of Wisconsin, USA

Institute for Plasma Research, University of Maryland, USA

Librarian, Fusion Energy Division, Oak Ridge National Laboratory, USA

Librarian, Institute of Fusion Studies, University of Texas, USA

Librarian, Magnetic Fusion Program, Lawrence Livermore National Laboratory, USA

Library, General Atomics, USA

Plasma Physics Group, Fusion Energy Research Program, University of California at San Diego, USA

Plasma Physics Library, Columbia University, USA

Alkesh Punjabi, Center for Fusion Research and Training, Hampton University, USA

Dr. W.M. Stacey, Fusion Research Center, Georgia Institute of Technology, USA

Dr. J ohn Willis, U.S. Department of Energy, Office of Fusion Energy Sciences, USA

Mr. Paul H. Wright, Indianapolis, Indiana, USA 
The Princeton Plasma Physics Laboratory is operated by Princeton University under contract with the U.S. Department of Energy.

\author{
Information Services \\ Princeton Plasma Physics Laboratory \\ P.O. Box 451 \\ Princeton, NJ 08543
}

Phone: 609-243-2750

Fax: 609-243-2751

e-mail: pppl_info@pppl.gov

Internet Address: http://www.pppl.gov 